\title{
Intergrating psychiatric nosology: from symptoms to syndromes
} George Alevizopoulos

\author{
Address: Community Mental Health Centre, Faculty of Nursing, School of Health Sciences, University of Athens, Greece \\ from International Society on Brain and Behaviour: 3rd International Congress on Brain and Behaviour \\ Thessaloniki, Greece. 28 November - 2 December 2007 \\ Published: 17 April 2008 \\ Annals of General Psychiatry 2008, 7(Suppl I):S57 doi:I0.II86/I744-859X-7-SI-S57
}

This abstract is available from: http://www.annals-general-psychiatry.com/content/7/SI/S57

(c) 2008 Alevizopoulos; licensee BioMed Central Ltd.

Psychiatric disorders represent the most complicated expression of human behaviour. Psychiatric phenomenology has been described in details showing pivotal differences among various psychiatric syndromes. However, in daily practice, the same medications are used to treat different disorders. This fascination invites a challenge: a need to describe high level, complex behavioural impairments in terms of underlying neuroanatomy, neurophysiology and neurochemistry. The introduction and refinement of the advanced functional neuroimaging techniques has reinvigorated the exploration of the neuronal bases for normal behaviour and emotion. These developments, together with improvements in structural imaging and rapid advances in a number of neuroscientific fields, have played a major part, over the last few decades, in re-establishing the concept of the brain in psychiatry.

Current research re-evaluates models of mental illness in response to the apparently galloping progress of neuroscience. It seeks to assemble 'a conceptual framework for understanding the mechanisms by which mental symptoms are generated'. After all, only a tiny minority of the psychological processes that govern even normal, relatively automatic, behaviour have been mapped with the remotest degree of completeness or confidence. In reality, therefore, any current attempt to establish 'the neural basis of mental symptoms', by establishing theoretical expressions of hallucinations, delusions, thought disorder, anxiety, depression, and so forth, in terms that resonate with those of basic neuroscience will be welcomed.

This presentation reviews the literature of the common underlying mechanisms suggested to govern major psychiatric syndromes. The pure clinician is likely to find this effort odd, but it helps to establish or enlarge a knowledge base that encourages forays away from purely clinical journals into the areas of basic science that are becoming increasingly relevant and, one thinks and wishes, indispensable, to the practice of psychiatry. 